\title{
Electrochemical studies of redox probes in self-organized lyotropic liquid crystalline systems ${ }^{\dagger}$
}

\author{
P SURESH KUMAR and V LAKSHMINARAYANAN* \\ Raman Research Institute, C.V. Raman Avenue, Sadashivanagar, Bangalore 560080 \\ e-mail: narayan@rri.res.in
}

\begin{abstract}
Lyotropic liquid crystalline phases formed by surfactants are of special importance due to their close resemblance to biological systems. The redox reactions in such ordered media are of fundamental interest in understanding several complex processes occurring in the biological media, where the former can act as model systems. In this work, we have carried out the redox reactions of benzoquinone|hydroquinone, methyl viologen and ferrocenemethanol probes in a lyotropic hexagonal columnar phase (H1 phase) using cyclic voltammetry and electrochemical impedance spectroscopic studies. The liquid crystalline phase we have studied is made up of the non-ionic surfactant, Triton X-100 and water. Polarizing optical microscopic examination confirmed that the columnar hexagonal phase is retained even after the addition of redox probe as well as the supporting electrolyte. Our studies show a significant shift in the half-peak potentials of the redox probes in the $\mathrm{H} 1$ phase as compared to the solvent phase. The diffusion coefficient values for different redox probes in the H1 phase were also found to be significantly reduced when compared to the corresponding solvent media.
\end{abstract}

Keywords. Lyotropic liquid crystal; redox reactions; half-peak potential; diffusion coefficient.

\section{Introduction}

Lyotropic liquid crystals (LLC) formed by the surfactants are of wide interest owing to the fact that they possess the orientational order of the crystalline phase as well as the dynamic nature of the liquid phase. ${ }^{1}$ Liquid crystalline phases formed by the surfactant molecules include nematic, smectic, columnar, lamellar, and cubic phases. ${ }^{2}$ These different phases are formed as a function of both the surfactant concentration as well as the temperature. The lyotropic liquid crystals especially the lamellar phase, resembles the biological medium since many of the lipids in the living system are shown to exhibit the lamellar phase. ${ }^{3}$ The LLCs can be model systems to understand the basic processes in biological and related media. These systems have also been widely used for the biosensor and drug delivery applications. $^{4,5}$

The non-ionic surfactant Triton X-100 has widely been used for the formation of LLCs. ${ }^{6-8}$ One of the major advantages of Triton X-100 is that it is nonionic in nature and so the interference of other ions, including the counter ions of the surfactants, can be

\footnotetext{
${ }^{\dagger}$ Dedicated to the memory of the late Professor S K Rangarajan *For correspondence
}

avoided in these systems. Bayer was the first to report the phase behaviour of the Triton X-100/water system. ${ }^{9}$ The studies show that the surfactant forms a columnar hexagonal phase ( $\mathrm{H} 1$ phase) in a wide range of surfactant concentration of about 40 to $60 \%$ by weight in the aqueous medium even at room temperature. Under conditions of high surfactant concentration and low temperature, it also forms a lamellar phase. The $\mathrm{Hl}$ phase of Triton X-100/water system has been explored for different applications. Previously we have studied the electrodeposition of nickel in this medium and have shown that this results in the formation of high surface area Ni deposits. ${ }^{10}$ This phase also finds application as a good medium for the formation of alkanethiol monolayers on the gold surface, where the electron barrier properties of these monolayers were shown to be better compared to the monolayers formed in the typical solvent media. ${ }^{11}$ We have also shown that thiol stabilized gold nanoparticles can be well dispersed in this medium without altering the hexagonal columnar order of the system. ${ }^{12}$ The H1 phase was also shown to be capable of dispersing carbon nanotubes. ${ }^{13}$

The electron transfer reactions of redox probes provide a simple method for the characterization of the lyotropic liquid crystalline phases and also for 
mimicking the biological electron transfer reactions. The redox reactions of different probes were studied in the lyotropic liquid crystalline phases by several groups. ${ }^{4,14-23}$ The diffusion coefficient of all these redox probes was found to decrease in the LLC media as compared to the solvent media. The extent of decrease was shown to depend on the nature of the redox probe as well as the nature of the medium. Bilewicz et al have explored the cubic phase of monoolein/water system for the immobilization of cholesterol oxidaze and other enzymes and have shown that the cubic phase provides an ideal medium for the redox reactions. They have also shown that the diffusion of different redox probes in the cubic phase was decreased compared to the solvent phase ${ }^{16-19}$ Kostela et al have reported the redox reactions of different redox probes in cubic as well as in the lamellar phase by using cyclic voltammetry and chronocoulometry. Their studies show that the redox potential values shift in the liquid crystalline phase compared to the solvent phase. Moreover, the diffusion coefficient of the systems was shown to be lesser in the liquid crystalline media. $^{21-23}$

In our previous report, the electron transfer studies using different redox probes, hexacyanoferrate (II|III) $\left[\mathrm{Fe}(\mathrm{CN})_{6}\right]^{4-\mid 3-}$, hexaammineruthenium(II|III) chloride $\left[\mathrm{Ru}\left(\mathrm{NH}_{3}\right)_{6}\right]^{2+13+}$ as well as ferrocene | ferrocenium $\mathrm{Fc} \mid \mathrm{Fc}^{+}$, have been carried out in the $\mathrm{Hl}$ phase of Triton X-100/water system. ${ }^{24}$ We showed that the half-peak potential values shift for different redox probes in the $\mathrm{H} 1$ phase as compared to the solvent phase. The shift in the redox potentials were shown to depend on the nature of the redox probes. The diffusion coefficient of all the redox probes was shown to be lower in the $\mathrm{H} 1$ phase when compared to the solvent phase. In the present report, we have carried out a detailed study of electrochemical processes using three redox probes, viz. benzoquinone hydroquinone, methyl viologen, and ferrocenemethanol in the columnar hexagonal phase $(\mathrm{H} 1$ phase) of Triton X-100/water system. Among them, benzoquinone is hydrophobic while the reduced species hydroquinone is hydrophilic and is known to form hydrogen bonds. Methyl viologen is a linear molecule and is a well-studied redox probe in the cubic phases. Ferrocenemethanol is amphiphilic in nature due to the hydrophobic ferrocene core and hydrophilic hydroxyl group. These molecules may occupy at the interface of the cylindrical micelles. We report in this manuscript the result of our study on these systems.

\section{Experimental section}

\subsection{Chemicals}

4-Benzoquinone (Lancaster), 4-hydroquinone (Loba Chemie), Methyl viologen (Aldrich), ferrocenemethanol (Aldrich), sodium fluoride (Merck), tetrabutylammonium tetrafluoroborate, TBATFB (Alfa Aeser) and propylene carbonate (Spectrochem) were used as received. The structures of the redox probes are presented in figure 1. Triton X-100 was obtained from Aldrich. Millipore water having a resistivity of $18 \mathrm{M} \Omega . \mathrm{cm}$ was used for the preparation of all aqueous solutions and the $\mathrm{H} 1$ phase.

\subsection{Electrode preparation}

Gold disc electrode of area $0.002 \mathrm{~cm}^{2}$ was used as the working electrode in all the experiments. Prior to each of the experiments, the electrode was polished with $1 \mu \mathrm{m}, 0.3 \mu \mathrm{m}$ and $0.05 \mu \mathrm{m}$ alumina slurries successively, sonicated for $2 \mathrm{~min}$, followed by treating with dilute aquaregia. The electrode was then washed with distilled water and rinsed with millipore water. The electrochemical experiments were carried out immediately after the electrode treatment.

\subsection{Electrolyte preparation}

The samples were prepared by following the same procedure as reported earlier. ${ }^{24}$ Briefly the aqueous solutions were made with $1 \mathrm{mM}$ concentration of the redox probes and $0 \cdot 1 \mathrm{M}$ concentration of supporting electrolyte, $\mathrm{NaF}$ in both aqueous as well as $\mathrm{Hl}$ phase. The columnar hexagonal phase ( $\mathrm{Hl}$ phase) has the composition of $42 \mathrm{wt} \%$ Triton X-100 and

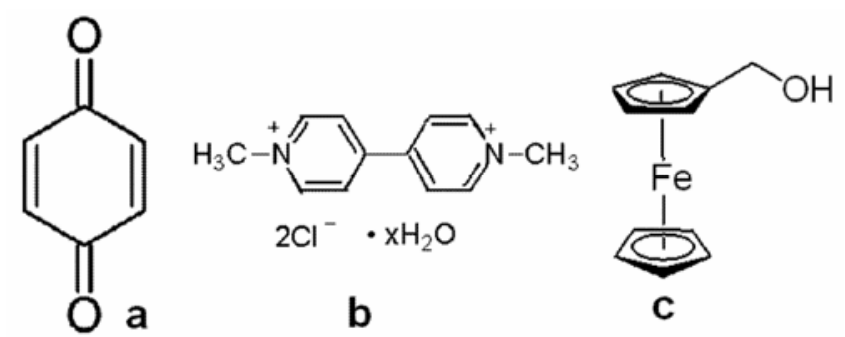

Figure 1. The chemical structures of (a) benzoquinone (b) methyl viologen and (c) ferrocenemethanol. 

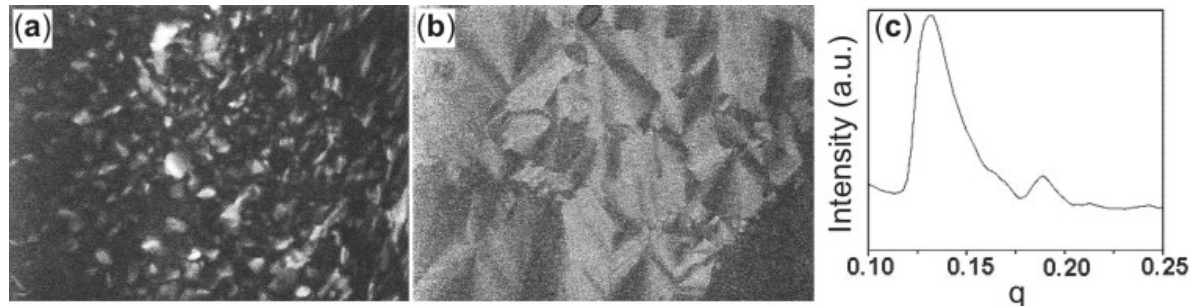

Figure 2. The polarizing optical micrographic textures of the H1 phase with (a) $1 \mathrm{mM}$ benzoquinone/hydroquinone and $0 \cdot 1 \mathrm{M} \mathrm{NaF}$, (b) $0 \cdot 1 \mathrm{M}$ TBATFB and $1 \mathrm{mM}$ ferrocenemethanol and (c) small angle X-ray scattering data for the $1 \mathrm{mM}$ methyl viologen with $0 \cdot 1 \mathrm{M} \mathrm{NaF}$ in the $\mathrm{H} 1$ phase.

$58 \mathrm{wt} \%$ water. For the preparation of the electrolytes in the $\mathrm{Hl}$ phase, the aqueous solution of the redox probes and the supporting electrolyte was made, which was then used for the preparation of $\mathrm{Hl}$ phase. The surfactant was then mixed well with the aqueous solution of the electrolytes at around $35^{\circ} \mathrm{C}$ and sonicated for $10 \mathrm{~min}$. The samples were then allowed to cool down to room temperature, which resulted in the formation of the $\mathrm{H} 1$ phase. The $\mathrm{H} 1$ phase is stable at room temperature and the columnar to isotropic transition occurs at around $30^{\circ} \mathrm{C}$. It was observed that higher concentration of the electrolytes results in the destabilization of the $\mathrm{Hl}$ phase. Ferrocenemethanol was studied in propylene carbonate medium as it is not soluble in water. $0 \cdot 1 \mathrm{M}$ TBATFB was used as the supporting electrolyte. For the preparation of $\mathrm{Hl}$ phase with ferrocenemethanol, it was dispersed in water with TBATFB and the dispersion was used for the preparation of $\mathrm{Hl}$ phase.

\subsection{Polarizing optical microscopy and small angle $X$-ray scattering}

The polarizing optical micrographs were obtained using an Olympus POM instrument. The textures were taken by sandwiching the samples in between glass slide and cover slips. All the images were taken at the room temperature. Small angle X-ray Scattering (SAXS) was carried out using an X-ray diffractometer (Rigaku UltraX 18) operating at $50 \mathrm{kV}$ and $80 \mathrm{~mA}$ using $\mathrm{CuK} \alpha$ radiation having a wavelength of $1.54 \AA$. Samples were filled in the capillary tube (Hampton research, USA) and sealed. All the scattering studies were carried out at room temperature.

\subsection{Electrochemical studies}

2.5a Cyclic voltammetry: A model 263A potentiostat (EG\&G) was used for the cyclic voltammetric studies. A single cell with a three-electrode system was used for the electrochemical studies with a saturated calomel electrode (SCE) as the reference electrode and platinum foil as the auxiliary electrode. In propylene carbonate, a silver wire electrode was used as a quasi-reference electrode, the potential of which was converted to that of SCE. All the plots shown are for the third cycle of the cyclic voltammogram.

\section{$2.5 \mathrm{~b}$ Electrochemical impedance spectroscopy} (EIS): EIS was performed using a model 5210 lock-in-amplifier (Perkin-Elmer Instruments) with Power Suite software $(E G \& G)$ interfaced with a PC. The impedance measurements were carried out over a wide frequency range of $100 \mathrm{mHz}$ to $100 \mathrm{kHz}$ and at an ac amplitude of $10 \mathrm{mV}$ at the formal potential or halfpeak potential as the case may be for the redox system. The impedance data were analysed using ZSimpWin software (EG\&G). All the electrochemical experiments were carried out at room temperature.

\section{Results and discussions}

\subsection{Polarizing optical microscopic (POM) studies}

Polarizing optical microscopy provides an easy assessment of the liquid crystalline phases. Figure $2 \mathrm{a}$ and $\mathrm{b}$ show the representative POM textures of quinone and ferrocenemethanol systems respectively in the $\mathrm{H} 1$ phase. The images resemble that of pure Triton X-100/water system indicating that even after the addition of the redox probe and the supporting electrolyte, the liquid crystalline phase ( $\mathrm{H} 1$ phase) is maintained. A detailed study of the systems using small-angle X-ray scattering (SAXS) confirm the retention of the $\mathrm{Hl}$ phase as the peaks in figure 2c follows the ratio $1: \sqrt{3}: \sqrt{4}$ which is typical for the H1 phase. The $d$-spacing corresponding to the first 
peak were calculated to be $52 \AA$, which is in accordance with the value for the pure Triton X-100/ water system. ${ }^{12}$

\subsection{Electrochemical studies}

We have carried out the cyclic voltammetry and electrochemical impedance spectroscopy studies in the solvent and in the $\mathrm{Hl}$ phase using the three different redox probes. The CV studies show that the half-peak potential shifts in the $\mathrm{H} 1$ phase compared to the solvent media. The EIS studies reveal that the kinetics and diffusion coefficient parameters of the system in both the media.

3.2a Benzoquinone hydroquinone system: Figure 3 shows the cyclic voltammograms of $1 \mathrm{mM}$ benzoquinone $+1 \mathrm{mM}$ hydroquinone in the $\mathrm{H} 1$ phase with $0 \cdot 1 \mathrm{M} \mathrm{NaF}$ as the supporting electrolyte. The inset of the figure shows the CV of the same system in the aqueous phase. The curves show that the redox reactions are under diffusion controlled regime both in the aqueous phase as well as in the $\mathrm{Hl}$ phase. It is clear from the comparison of the CVs that the peak currents have reduced to a large extent in the $\mathrm{H} 1$ phase when compared to the aqueous phase. The system shows two redox peaks in accordance with the reported mechanism of a two electron transfer reaction as given below: ${ }^{25}$

$$
\begin{aligned}
& \mathrm{Bq}^{2-} \rightarrow \mathrm{Bq}^{-}+e^{-}, \\
& \mathrm{Bq}^{-} \rightarrow \mathrm{Bq}+e_{-}^{-} .
\end{aligned}
$$

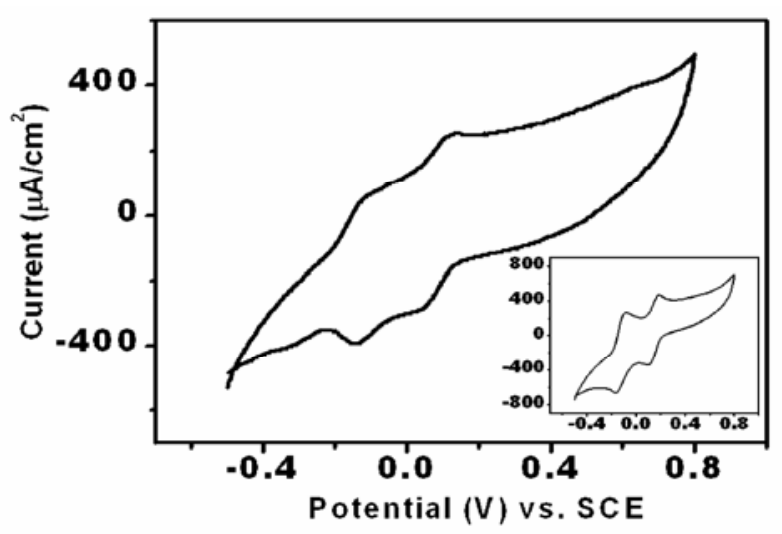

Figure 3. Cyclic voltammogram for the $1 \mathrm{mM}$ Benzoquinone|Hydroquinone/0.1 M NaF system in the $\mathrm{H} 1$ phase. The inset shows the cyclic voltammogram for the same redox system in the aqueous phase.
The peaks corresponding to the reaction (1) is rather broad and hence not discussed further. The potentials corresponding to the reaction (2) in both the phases are presented in table 1. As clear from the table, the system undergoes a quasi-reversible redox

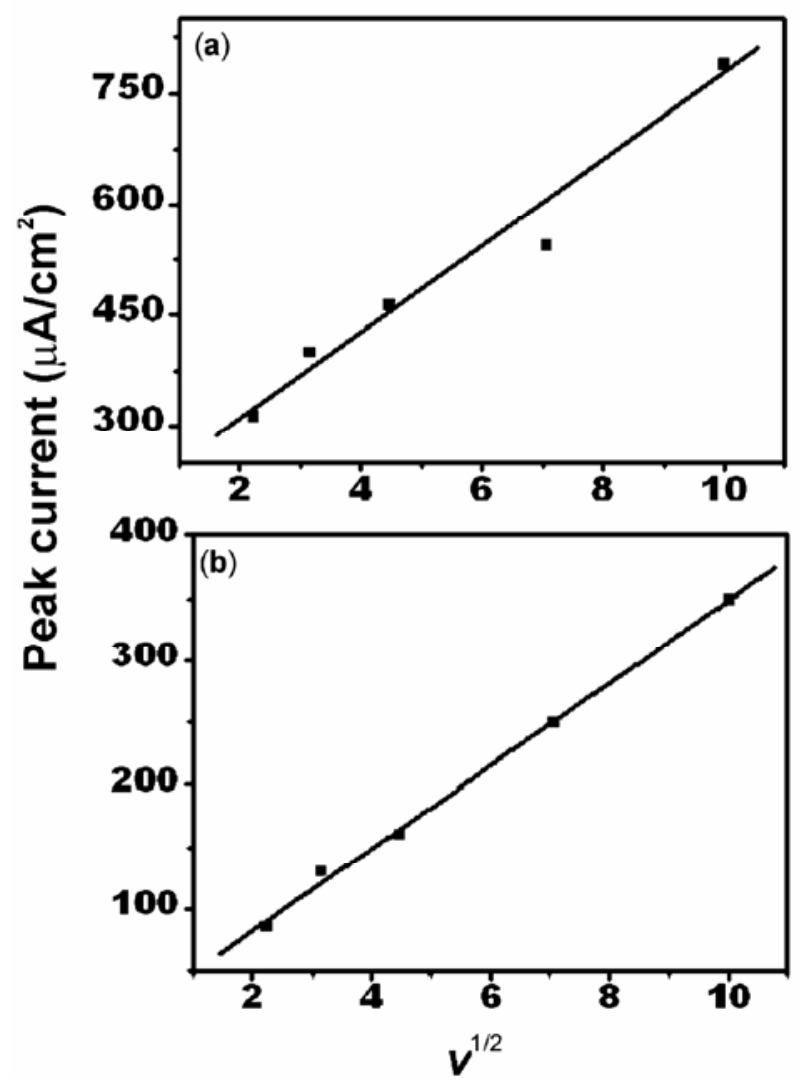

Figure 4. Plots of peak current vs square root of scan rate, $v^{1 / 2}$ for the $1 \mathrm{mM}$ benzoquinone/hydroquinone/ $0 \cdot 1 \mathrm{M} \mathrm{NaF}$ system (a) in the aqueous phase and (b) in the H1 phase.

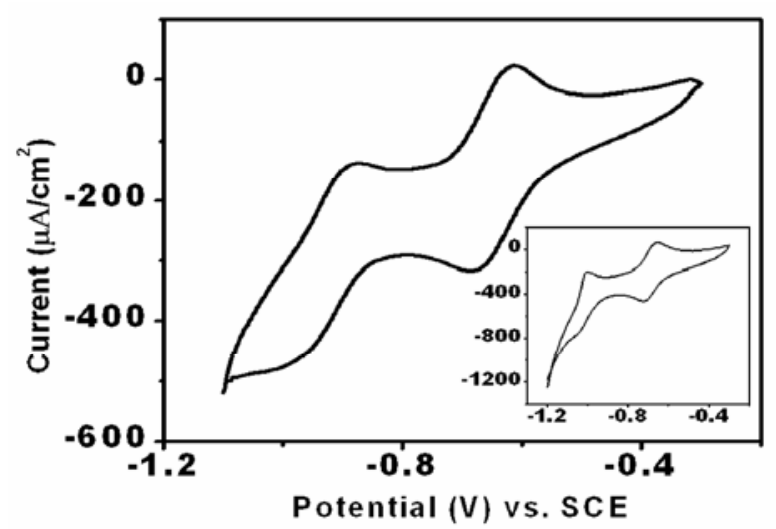

Figure 5. Cyclic voltammogram for the $1 \mathrm{mM}$ methyl viologen/0.1 M NaF system in the $\mathrm{H} 1$ phase. The inset shows the cyclic voltammogram for the same redox system in the aqueous phase. 
Table 1. Anodic and cathodic peak potential $\left(E_{\mathrm{p}}^{\mathrm{a}}\right.$ and $\left.E_{\mathrm{p}}^{\mathrm{c}}\right)$, peak separation $\left(\Delta E_{\mathrm{p}}\right)$, and half-peak potential $\left(E_{1 / 2}\right)$ values for the $1 \mathrm{mM}$ benzoquinone $1 \mathrm{mM}$ hydroquinone redox reaction using $0 \cdot 1 \mathrm{M} \mathrm{NaF}$ as the supporting electrolyte.

\begin{tabular}{lccccc}
\hline Medium & Scan rate $(\mathrm{mV})$ & $E_{\mathrm{P}}^{\mathrm{a}}(\mathrm{mV})$ & $E_{\mathrm{P}}^{\mathrm{c}}(\mathrm{mV})$ & $\Delta E_{P}(\mathrm{mV})$ & $E_{1 / 2}(\mathrm{mV})$ \\
\hline Aqueous phase & 10 & 180 & 95 & 85 & 138 \\
& 20 & 184 & 120 & 64 & 152 \\
& 50 & 184 & 101 & 83 & 143 \\
\multirow{4}{*}{ H1 phase } & 100 & 190 & 96 & 94 & 143 \\
& 10 & 112 & 41 & 81 & 75 \\
& 20 & 124 & 50 & 74 & 87 \\
& 50 & 135 & 33 & 102 & 84 \\
& 100 & 131 & 34 & 97 & 83 \\
\hline
\end{tabular}

Table 2. Anodic and cathodic peak potential $\left(E_{\mathrm{p}}^{\mathrm{a}}\right.$ and $\left.E_{\mathrm{p}}^{\mathrm{c}}\right)$, peak separation $\left(\Delta E_{\mathrm{p}}\right)$, and half-peak potential $\left(E_{1 / 2}\right)$ values for the $1 \mathrm{mM}$ methyl viologen redox reaction using $0 \cdot 1 \mathrm{M} \mathrm{NaF}$ as the supporting electrolyte.

\begin{tabular}{lccccc}
\hline Medium & Scan rate $(\mathrm{mV})$ & $E_{\mathrm{p}}^{\mathrm{a}}(\mathrm{mV})$ & $E_{\mathrm{p}}^{\mathrm{c}}(\mathrm{mV})$ & $\Delta E_{P}(\mathrm{mV})$ & $E_{1 / 2}(\mathrm{mV})$ \\
\hline Aqueous phase & 10 & -653 & -715 & 62 & -684 \\
& 20 & -654 & -718 & 64 & -686 \\
& 50 & -654 & -714 & 60 & -684 \\
\multirow{3}{*}{ H1 phase } & 100 & -651 & -719 & 68 & -685 \\
& 10 & -616 & -676 & 60 & -646 \\
& 20 & -608 & -670 & 62 & -639 \\
& 50 & -612 & -678 & 66 & -645 \\
& 100 & -614 & -676 & 62 & -645 \\
\hline
\end{tabular}

reaction in both aqueous and $\mathrm{Hl}$ phases with a peak separation of $80 \mathrm{mV}$. The important point to be noted from the table is the shift in the half-peak potentials in the $\mathrm{Hl}$ phase as compared to the aqueous phase. For example, at the scan rate of $50 \mathrm{mV} / \mathrm{s}$, the $E_{1 / 2}$ in the aqueous phase is $143 \mathrm{mV}$ while in the $\mathrm{H} 1$ phase, it has shifted to $84 \mathrm{mV}$. In other words, the $E_{1 / 2}$ has shifted to more negative potential in the $\mathrm{H} 1$ phase. This can be explained in terms of the stability of the redox probes in the Hl medium. The reduction of benzoquinone in a non-buffered solution results in the formation of different reduced species like $\mathrm{Bq}^{2-}, \mathrm{BqH}^{-}$as well as $\mathrm{BqH}_{2}$ as reported by Quan and coworkers. ${ }^{22}$ It is known that the hydroquinone is more hydrophilic compared to the benzoquinone. The redox reaction of the quinone system results in a transition between hydrophobic benzoquinone and hydrophilic hydroquinone. In the H1 phase, benzoquinone is stabilized inside the core of the cylindrical micelle by the hydrophobic interactions, which is not possible in the aqueous phase. Moreover, hydroquinone can form hydrogen bonds with the water molecules outside the micelle. In the $\mathrm{Hl}$ phase, most of the water molecules are bound to the micelles which make a less hydrated reduced species. As a result, the energy cost of breaking the hydrogen bond of hydroquinone during oxidation is lesser in the $\mathrm{Hl}$ phase compared to the aqueous phase. In other words, the oxidation is more favoured in the $\mathrm{Hl}$ phase compared to the aqueous phase and the half peak potential shifts to a more negative value in the $\mathrm{H} 1$ phase.

Figure 4 shows the plot of the peak current vs the square root of scan rate. The linearity of the plot over the wide range of scan rates shows that in the H1 phase, the redox reaction take place under a linear diffusion regime. Even though the linearity may not indicate the reversibility of the redox reactions, it points to the fact that the reactions in the $\mathrm{H} 1$ phase using benzoquinone|hydroquinone follows the Randles-Sevcik equation. ${ }^{26}$ Further studies on the mass transport properties have been carried out using electrochemical impedance spectroscopy which is discussed later.

3.2b Viologen system: Figure 5 shows the $\mathrm{CV}$ of methyl viologen system in the $\mathrm{Hl}$ phase. The $\mathrm{CV}$ of the same system in the aqueous phase is shown in 
the inset of the figure. The peak potentials for the system at different scan rates are shown in the table 2 . The peak separation in both the media is about $65 \mathrm{mV}$. Two redox peaks have been observed for the methyl viologen $(\mathrm{mv})$ corresponding to the two electron transfer processes in accordance with the previous reports. ${ }^{20}$ This corresponds to the two oneelectron transfer reactions, that is

$$
\begin{aligned}
& m v \rightarrow m v^{+}+e^{-}, \\
& m v^{+} \rightarrow m v^{2+}+e^{-}
\end{aligned}
$$

A further examination of table 2 reveals that the half-peak potentials have shifted to a more positive value in the $\mathrm{H} 1$ phase compared to the aqueous phase. At the scan rate of $50 \mathrm{mV} / \mathrm{s}$, the $E_{1 / 2}$ for the viologen system in the $\mathrm{H} 1$ phase is $-645 \mathrm{mV}$ compared to the value in the aqueous phase of $-684 \mathrm{mV}$. This is contrary to the observation in the case of benzoquinone|hydroquinone where the $E_{1 / 2}$ has been shifted to a more negative potential. However, the positive shift in this case is less as compared to the negative shift for the benzoquinone/hydroquinone system. The positive half-peak potential shift of viologen system may be explained in what follows. As mentioned above, the oxidation of the methyl viologen results in the mono and dicationic species. The oxidation increases the net charge on the redox probe. In the $\mathrm{Hl}$ phase, most of the water molecules are bound to the surfactant molecules to form the cylindrical micelles of the columnar phase. As a result, the solvation energy for the oxidized species is less in the $\mathrm{H} 1$ phase compared to the aqueous phase. For the solvation, the hydrogen bonding between the water molecules and the surfactants has to be broken. As a result the oxidized species is less stable in the $\mathrm{H} 1$ phase and so the oxidation reaction is less favoured. Further, due to the hydrophobic nature of the methyl viologen stays inside the cylindrical micelles of Triton X-100/water system. This makes the oxidized species, which is hydrophilic to be less favoured. As a result of this, the oxidation needs more energy and the half-peak potential shifts to more positive value.

Figure 6 shows the plot of peak current vs. square root of scan rate for the viologen system. Figure $6 \mathrm{a}$ shows the plot for the aqueous medium while figure $6 \mathrm{~b}$ presents the plot for the $\mathrm{H} 1$ phase. It is clear from the plots that the peak current varies linearly with the square root of scan rate. This indicates that the viologen system also follows the Randles Sevcik equation both in the aqueous as well as in the H1 phase.

3.2c Ferrocenemethanol system: Figure 7 shows the cyclic voltammogram of the ferrocenemethanol system in the $\mathrm{Hl}$ phase using $0 \cdot 1 \mathrm{M}$ TBATFB as the

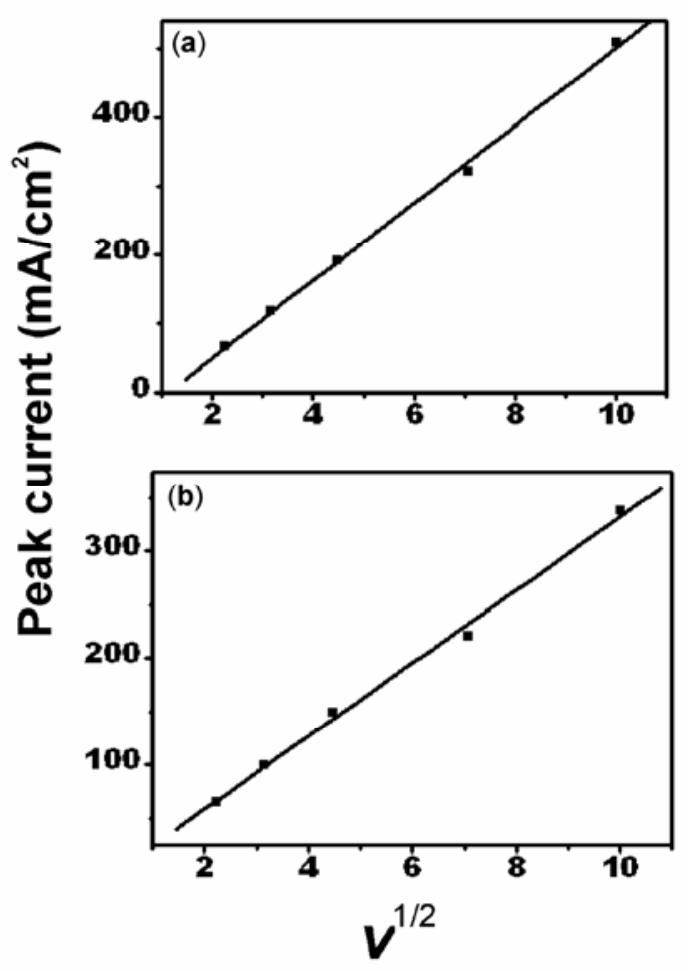

Figure 6. Plots of peak current vs square root of scan rate, $v^{1 / 2}$ for the $1 \mathrm{mM}$ methyl viologen/0.1 M NaF (a) in the aqueous phase and (b) in the H1 phase.

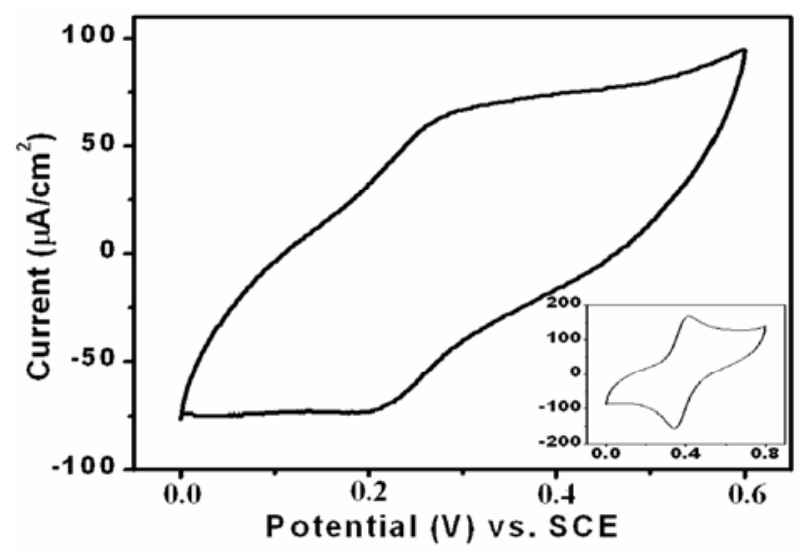

Figure 7. Cyclic voltammogram for the $1 \mathrm{mM}$ ferrocenemethanol/0.1 M NaF system in the $\mathrm{H} 1$ phase. The inset shows the cyclic voltammogram for the same redox system in the propylene carbonate medium. 
Table 3. Anodic and cathodic peak potential $\left(E_{\mathrm{p}}^{\mathrm{a}}\right.$ and $\left.E_{\mathrm{p}}^{\mathrm{c}}\right)$, peak separation $\left(\Delta E_{\mathrm{p}}\right)$, and half-peak potential $\left(E_{1 / 2}\right)$ values for the $1 \mathrm{mM}$ ferrocenemethanol redox reaction using $0 \cdot 1 \mathrm{M}$ TBATFB as the supporting electrolyte.

\begin{tabular}{lccccc}
\hline System & $\begin{array}{c}\text { Scan rate } \\
v(\mathrm{mV} / \mathrm{s})\end{array}$ & $E_{\mathrm{p}}^{\mathrm{a}}(\mathrm{mV})$ & $E_{\mathrm{p}}^{\mathrm{c}}(\mathrm{mV})$ & $\Delta E_{\mathrm{p}}(\mathrm{mV})$ & $E_{1 / 2}(\mathrm{mV})$ \\
\hline Propylene & 10 & 552 & 488 & 64 & 520 \\
carbonate medium & 20 & 554 & 494 & 60 & 524 \\
& 50 & 578 & 500 & 78 & 539 \\
H1 phase & 100 & 580 & 490 & 90 & 535 \\
& 10 & 286 & 218 & 68 & 252 \\
& 20 & 292 & 210 & 82 & 251 \\
& 50 & 296 & 198 & 98 & 247 \\
& 100 & 296 & 196 & 100 & 246 \\
\hline
\end{tabular}

supporting electrolyte. The inset of the figure shows the voltammogram of the same system in the propylene carbonate medium. Table 3 presents the redox potentials of the system in both media. The table shows that the half-peak potentials have shifted to a more negative value by $270 \mathrm{mV}$ in the $\mathrm{H} 1$ phase as compared to the propylene carbonate medium. This can be explained as follows. Ferrocenemethanol is hydrophobic in nature with the hydroxyl functionalization in the side chain. Therefore these molecules will prefer to extent the hydroxyl group to water molecules at the outer boundary of the $\mathrm{Hl}$ phase while the hydrophobic part of the molecules inside the core of the micelles. But the oxidized species is highly hydrophilic in nature and can occupy the inter-columnar space of the micelle. As a result, the energy cost in the reorganization of the ferrocenemethanol from inside the micelle to outside the micelle during the oxidation is less due to the more hydrophilic nature of the reduced species. Moreover, the oxidized species can form hydrogen bond with the water molecules, which can stabilize the ions. The oxidized species can also interact with the electron-rich polyethoxy chain of the surfactant. These two interactions along with the lesser reorganization energy result in a negative potential shift for the ferrocenemethanol system. This negative shift is in contrast to the positive halfpeak potential shift observed for ferrocene systems. ${ }^{24}$ For the ferrocene system, the reduced species occupy the space inside the micelle due to the hydrophobic nature and as a result the reorganization energy is more during the oxidation. Also the ferrocenium ions can not form hydrogen bonds with the water molecules. Due to these two reasons, the half-peak potential of the ferrocene system shifts to a more positive potential. The plot of peak current vs square root of scan rate for the ferrocenemethanol system is presented in figure 8 for the reaction in propylene carbonate medium and $\mathrm{Hl}$ phase. The linearity of the plot shows that the reaction is under planar diffusion controlled regime as expected by the Randles-Sevcik equation. ${ }^{26}$

\section{2d Electrochemical impedance spectroscopy:}

We have studied the impedance response of all the redox probes in the solvent as well as in the $\mathrm{H} 1$ phase for further information on the kinetics as well as diffusion characteristics of the redox probes. Figures 9,10 and 11 show the real and imaginary parts of the impedance for the benzoquinone| hydroquinone, methyl viologen and ferrocenemethanol systems. The plots reveal that the reaction is under diffusion control regime since it forms a semi-circle at high frequencies and a straight line with an angle close to $45^{\circ}$ at low frequencies. The semicircle represents the double layer capacitance and the kinetics of the reaction. The low frequency part represents the Warburg impedance, which relates to the diffusion of the probes. The impedance data were fitted with the simple Randles equivalent circuit, uncompensated resistance $(\mathrm{Ru})$ in series with the parallel combination of double-layer capacitance (Cdl) and charge transfer resistance (Rct) with Warburg impedance $(\mathrm{Zw})$ in series, that is $\mathrm{R}(\mathrm{C}(\mathrm{RW})$. Table 4 shows the fitting parameters for all the redox probes in the solvent as well as in the $\mathrm{Hl}$ phase. A detailed examination of the table shows that the solution resistance has increased in the $\mathrm{Hl}$ phase. This is due to the lower conductivity of the liquid crystalline medium as compared to the aqueous phase. Further, it shows that charge-transfer resis- 
tance $\left(R_{\mathrm{ct}}\right)$ values in the $\mathrm{Hl}$ phase has not increased much compared to the aqueous phase, which means that the kinetics of the reaction is still facile in the $\mathrm{H} 1$ phase. The Warburg impedance is observed to be more in the $\mathrm{Hl}$ phase indicating a slower diffusion of the probes in the $\mathrm{Hl}$ phase.

The diffusion coefficients of the redox probes both in the solvent and $\mathrm{H} 1$ phase were calculated by the following equations. ${ }^{26}$

$$
\begin{aligned}
& Z_{w}=(2 / \omega)^{1 / 2} \cdot \sigma \\
& \sigma=\left(R T / 2^{1 / 2} n^{2} F^{2} D^{1 / 2} A\right)\left(1 / C_{O}+1 / C_{R}\right),
\end{aligned}
$$

where $\omega$ is the angular frequency, $F$ the Faraday constant, $A$ the area of electrode, $n$ the number of

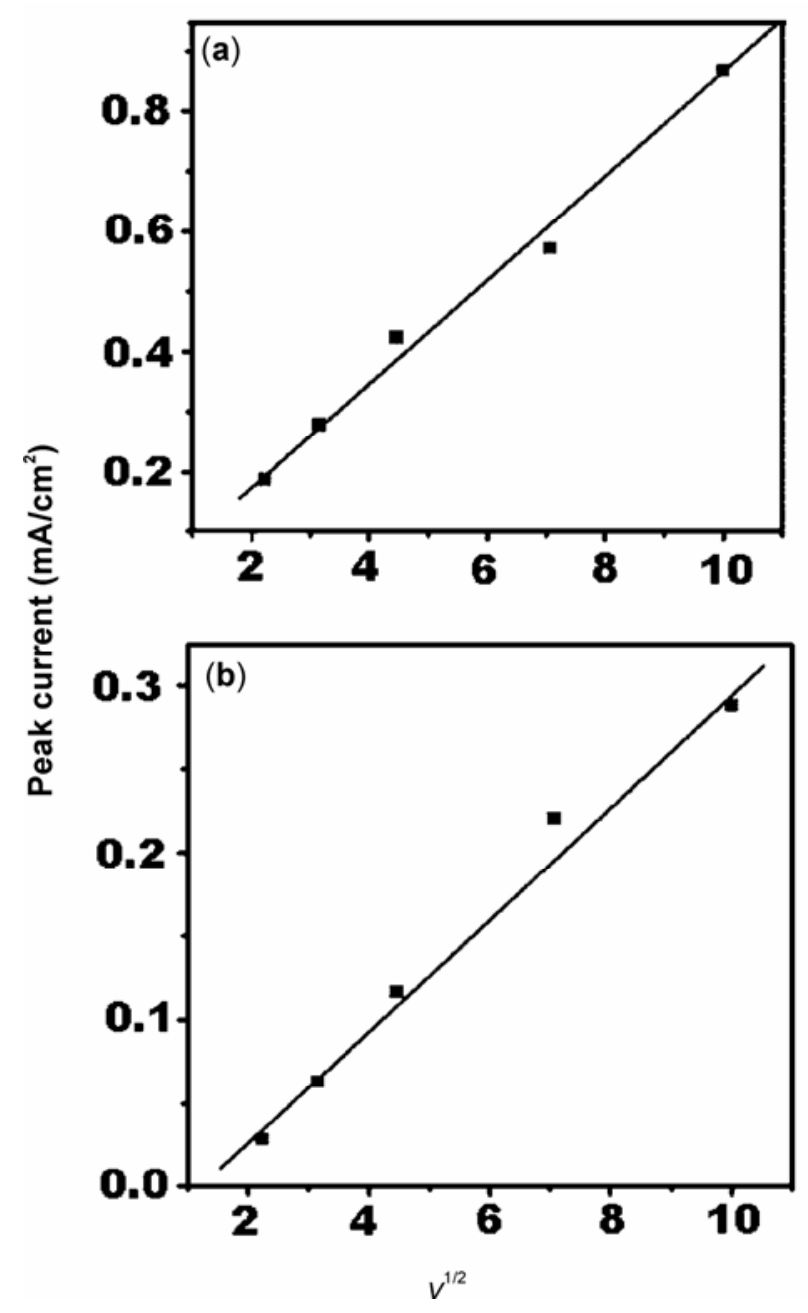

Figure 8. Plots of peak current vs square root of scan rate, $v^{1 / 2}$ for the $1 \mathrm{mM}$ ferrocenemethanol/0.1 M TBATFB system (a) in the propylene carbonate medium and (b) in the $\mathrm{H} 1$ phase. electrons involved, $D$ the apparent diffusion coefficient, $C_{\mathrm{O}}$ and $C_{\mathrm{R}}$ are the concentration of oxidized and reduced species. The calculated apparent diffusion coefficient values are presented in table 5 . It is clear that the $D_{\text {app }}$ has decreased in the $\mathrm{Hl}$ phase compared to the aqueous phase in accordance with other redox probes. The $D_{\text {app }}$ value has decreased almost by an order of magnitude for the benzoquinone/hydroquinone system whereas for the

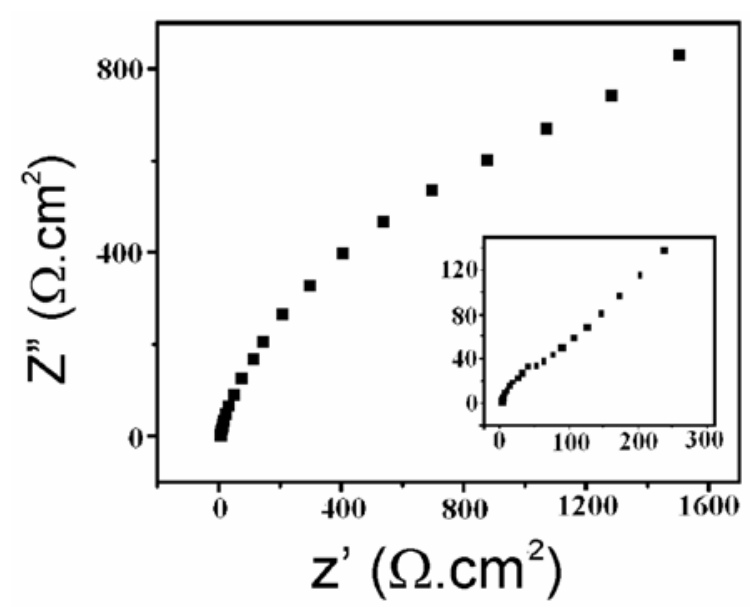

Figure 9. The real and imaginary part of the impedance for the redox reaction of $1 \mathrm{mM}$ benzoquinone| hydroquinone/0.1 M NaF system in the $\mathrm{H} 1$ phase. Inset: Impedance of the same redox system in the aqueous phase. Frequency range: $100 \mathrm{mHz}$ to $100 \mathrm{kHz}$, a.c. amplitude: $10 \mathrm{mV}$ peak to peak at the half-peak potential of the system.

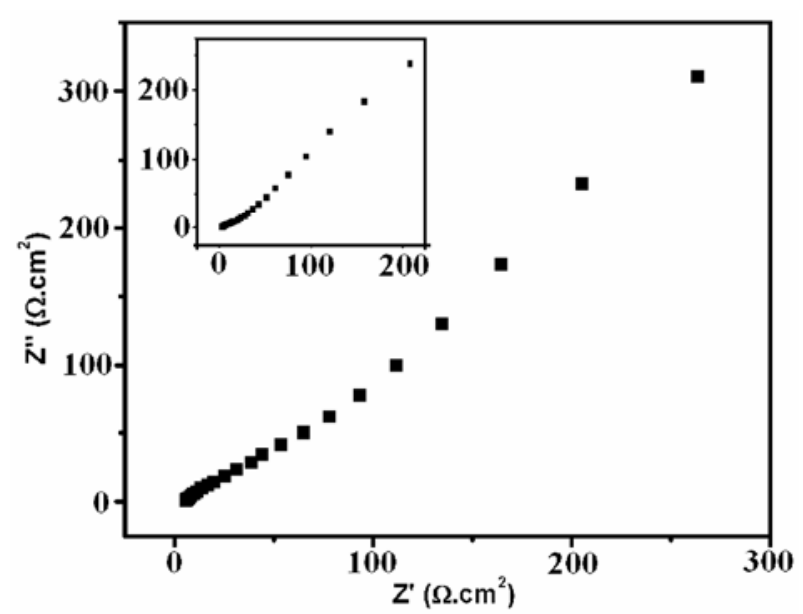

Figure 10. The real and imaginary part of the impedance for the redox reaction of $1 \mathrm{mM}$ methyl viologen/ $0 \cdot 1 \mathrm{M} \mathrm{NaF}$ system in the $\mathrm{H} 1$ phase. Inset: Impedance for the same redox system in the aqueous phase. Frequency range: $100 \mathrm{mHz}$ to $100 \mathrm{kHz}$, a.c. amplitude: $10 \mathrm{mV}$ peak to peak at the half-peak potential of the system. 
Table 4. Values of the solution resistance $\left(R_{\mathrm{u}}\right.$, in $\left.\Omega\right)$, charge-transfer resistance $\left(R_{\mathrm{ct}}\right.$ in $\left.\Omega . \mathrm{cm}^{2}\right)$, double layer capacitance $\left(C_{\mathrm{dl}}\right.$ in $\left.\mathrm{F} / \mathrm{cm}^{2}\right)$ and Warburg impedance $\left(Z_{\mathrm{w}}\right.$ in $\left.\mathrm{S} . \mathrm{s}^{1 / 2} . \mathrm{cm}^{2}\right)$, calculated by fitting the impedance data. *quinone and methyl viologen systems are studied in the aqueous medium while ferrocenemethanol is studied in propylene carbonate medium.

\begin{tabular}{|c|c|c|c|c|c|c|c|c|}
\hline & \multicolumn{4}{|c|}{ Solvent medium* } & \multicolumn{4}{|c|}{ H1 medium } \\
\hline & $R_{u}$ & $C_{d l}$ & $R_{c t}$ & $Z_{W}$ & $R_{u}$ & $C_{d l}$ & $R_{c t}$ & $Z_{W}$ \\
\hline $\mathrm{Bq}$ & 1123 & $1.884 \times 10^{-4}$ & 40 & $5 \cdot 1 \times 10^{-3}$ & 3191 & $1.1 \times 10^{-4}$ & $742 \cdot 8$ & $1.6 \times 10^{-2}$ \\
\hline MV & 823 & $1.192 \times 10^{-4}$ & 926 & $2 \cdot 2 \times 10^{-3}$ & 2591 & $5.9 \times 10^{-4}$ & $8 \cdot 155$ & $3.3 \times 10^{-3}$ \\
\hline $\mathrm{Fm}$ & 5270 & $1.515 \times 10^{-5}$ & 9 & $3 \cdot 3 \times 10^{-3}$ & $2.5 \times 10^{4}$ & $1.8 \times 10^{-5}$ & $66 \cdot 26$ & $5 \cdot 8 \times 10^{-4}$ \\
\hline
\end{tabular}

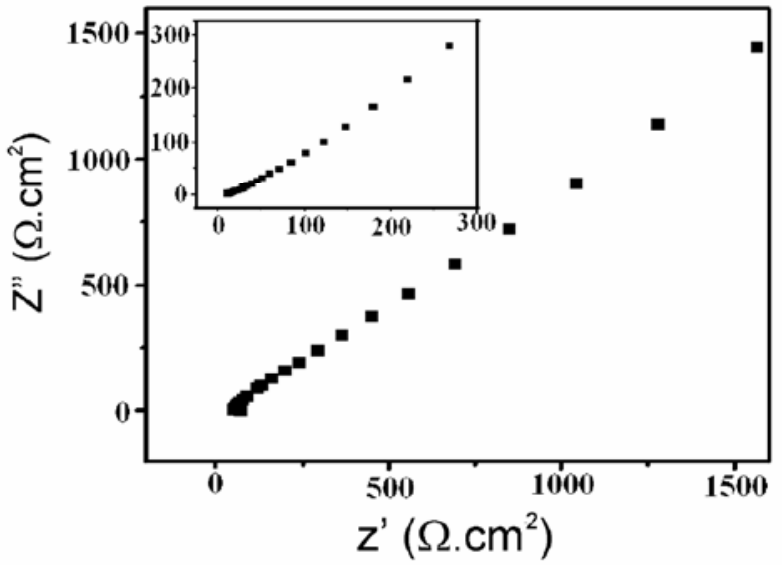

Figure 11. The real and imaginary part of the impedance for the redox reaction of the $1 \mathrm{mM}$ ferrocenemethanol/0.1 M TBATFB system in the H1 phase. Inset: Impedance for the same system in the propylene carbonate. Frequency range: $100 \mathrm{mHz}$ to $100 \mathrm{kHz}$, ac amplitude: $10 \mathrm{mV}$ peak to peak at the half-peak potential of the system.

methyl viologen system, it has reduced almost by 3 times. The large decrease in the $D_{\text {app }}$ of benzoquinone |hydroquinone can be explained in terms of the restricted mobility of the benzoquinone in the core of the $\mathrm{Hl}$ phase which is hydrophobic as compared to the outer aqueous phase. Moreover, hydroquinone can form hydrogen bonds with the surfactant-bounded water clusters, thereby reducing the diffusion of the species.

On the other hand, we have observed a very small decrease in the diffusion coefficient for the viologen system. This may probably be connected to the shape of the molecule. Unlike all other redox probes we have studied, methyl viologen is a rod-like molecule. The viologen molecules can thus move through the aqueous channels outside the micelle easily. This shows that the diffusion of the hydrophilic probes through the hydrophilic channels of the $\mathrm{Hl}$ phase is not hindered significantly. The SAXS studies (as shown in figure 2c) of the systems are also in agreement with this model, where we find that the $d$ spacing of the systems has not altered even after the dispersion of the redox probes as well as the supporting electrolyte.

The diffusion coefficient of ferrocenemethanol system in the propylene carbonate as well as in the $\mathrm{H} 1$ phase is presented in the table 5. It is clear from the table that the values are less by about 30 times in the $\mathrm{H} 1$ phase compared to the solvent phase. This is very similar to the behaviour of the ferrocene system, where we have observed a similar decrease in the diffusion coefficient in the $\mathrm{H} 1$ phase. This can be rationalized as due to the interaction of reduced species inside the micelle and the oxidized species with the polyethoxy chain and water molecules outside the micelle.

Interestingly, the behaviours of ferrocene and ferrocenemethanol are observed to be very different. For the ferrocene system, the half-peak potential was shifted to a more positive potential, while for the ferrocenemethanol it has shifted to a lower potential compared to propylene carbonate medium. This shows that side chain functionalization of the probes affect its diffusion dynamics in the medium and charge-transfer phenomena on the electrode. On the other hand, it is noted that the reversibility of the system decreases after functionalizing the probes. This is clear from the table 3 while comparing the peak potentials for ferrocenemethanol and ferrocene which was reported earlier. ${ }^{24}$

The table 6 shows the comparison of the ratio of peak currents measured using the $\mathrm{CV}$ and the ratio of square root of the diffusion coefficients, which are measured using electrochemical impedance method in aqueous and $\mathrm{Hl}$ phase. The reasonable agreement of these two ratios by two different methods again supports our assumption of planar diffusion controlled model for the redox reaction in both aqueous as well as in the $\mathrm{Hl}$ phase. 
Table 5. Calculated values of apparent diffusion coefficient $\left(D_{\text {app }}\right)$ of the redox probes (in $\mathrm{cm}^{2} / \mathrm{s}$ ), studied in the solvent medium and in the $\mathrm{H} 1$ phase.

\begin{tabular}{lcc}
\hline System & Solvent phase & H1 phase \\
\hline Quinone system & $3 \cdot 26 \times 10^{-6}$ & $8 \cdot 6 \times 10^{-8}$ \\
Viologen system & $5 \cdot 1 \times 10^{-6}$ & $2 \cdot 9 \times 10^{-6}$ \\
Ferrocenemethanol system & $3 \cdot 1 \times 10^{-6}$ & $9 \cdot 8 \times 10^{-8}$ \\
\hline
\end{tabular}

Table 6. Ratios of peak currents $\left(I_{\mathrm{p}}\right)$ measured using cyclic voltammetry and square root of apparent diffusion coefficient values $\left(D^{1 / 2}\right)$ calculated from electrochemical impedance spectroscopy, in the solvent phase and in the aqueous phase for the different redox probes.

\begin{tabular}{lcc}
\hline System & $I_{\mathrm{sol}} / I_{\mathrm{H} 1}$ & $D^{1 / 2}{ }_{\mathrm{sol}} / D^{1 / 2} \mathrm{Hl}$ \\
\hline Quinone system & 0.443 & 0.425 \\
Viologen system & 0.683 & 0.754 \\
Ferrocenemethanol system & $0 \cdot 2267$ & $0 \cdot 1778$ \\
\hline
\end{tabular}

The studies also bring several other issues like the orientation of the redox probes and the surfactant micelles near the electrode surface. In our studies, we have assumed that the effective area of the electrode in contact with the electrolyte remains the same in both the solvent phase as well as in the $\mathrm{Hl}$ phase which means that the effect of adsorption or the orientation of the surfactant molecules is not considered. But it is worth noting that the probes still undergo planar diffusion process, indicating that the access of the probes to the electrode is not hindered by the surfactant.

Prof. S K Rangarajan had a deep interest in several aspects of transport phenomenon in biological membranes. ${ }^{27}$ Further work using ac and nonlinear relaxation techniques ${ }^{28}$ using the redox probes will throw light on several interesting electrochemical processes in such model biological systems described here.

\section{Conclusion}

We have carried out electrochemical studies of three redox probes in the $\mathrm{H} 1$ phase of Triton X-100/water system. The cyclic voltammetry studies show that the stabilization of the redox species in the liquid crystalline medium plays a definite role on the $E_{1 / 2}$ potentials of the redox reactions. Further studies using electrochemical impedance studies show that the diffusion coefficient of the redox probes has decreased in the $\mathrm{Hl}$ phase. From the results, we infer that this medium has the potential to be used as model system for the biological electron transfer studies.

\section{References}

1. Imrie $\mathrm{C} T$ and Luckhurst G R 1998 In Handbook of liquid crystals (eds) D Demus, J Goodby, G W Gray, H-W Spiess and V Vill (Weinheim: Wiley-VCH) vol. 2B p. 801

2. Petrov A G 1999 In The lyotropic state of matter: Molecular physics and living matter physics (Gordon \& Breach Sci. Pub.)

3. Chernomordik L, Kozlov M M and Zimmerberg J 1995 J. Membrane Biol. 1461

4. Nazaruk E, Bilewicz R, Lindblom G and Sethson B L 2008 Anal. Bioanal. Chem. 3911569

5. Kossena G A, Charman W N, Boyd B J and Porter C J H 2004 J. Controlled Release 99217

6. Ahir S V, Petrov P G and Terentjev E M 2002 Langmuir 189140

7. Galatanu A N, Chronakis I S, Anghel D F A and Khan A 2000 Langmuir 164922

8. Maza A D L and Parra J L 1994 Biochem. J. 303907

9. Beyer K 1982 J. Colloid Interface Sci. 8673

10. Ganesh V and Lakshminarayanan V 2004 Electrochim. Acta 493561

11. Ganesh V and Lakshminarayanan V 2006 Langmuir 221561

12. Kumar P S, Pal S K, Kumar S and Lakshminarayanan V 2007 Langmuir 233445

13. Weiss V, Thiruvengadathan R and Regev O 2006 Langmuir 22854

14. Eggins B R and Chambers J Q $1970 \mathrm{~J}$. Electrochem. Soc. Electrochem. Sci. 117186

15. Lee C W, Kyung M and Jang J M 1993 Langmuir 9 1934

16. Rowinski P, Korytkowska A and Belewicz R 2003 Chem. Phys. Lipids 124147

17. Belewicz R, Rowinski P and Rogalska E 2005 Bioelectrochemistry 663

18. Rowinski P, Bilewicz R, Stebe M-J and Rogalska E 2002 Anal. Chem. 741554

19. Ropers M H, Bilewicz R, Stebe M J, Hamidi A, Miclo A and Rogalska E 2001 Phys. Chem. Chem. Phys. 3240

20. Dalton E F and Murray R W 1991 J. Phys. Chem. 95 6383

21. Kostela J, Elmgren M, Kadi M and Almgren M 2005 J. Phys. Chem. B109 5073

22. Kostela J, Elmgren M and Almgren M 2005 Electrochim. Acta $\mathbf{5 0} 3333$

23. Kostela J, Elmgren M, Hansson $\mathrm{P}$ and Almgren $\mathrm{M}$ 2002 J. Electroanal. Chem. 53697

24. Kumar P S and Lakshminarayanan V 2007 Langmuir 231548

25. Quan M, Sanchez D, Wasylkiw M F and Smith D K 2007 J. Am. Chem. Soc. 12912847

26. Bard A J and Faulkner L R 2004 In Electrochemical methods - fundamental and applications (New Delhi: John Wiley \& Sons (Asia) Pte Ltd) Chapter 6, 10

27. De Levie R, Rangarajan S K and Seelig P F 1979 Biophys. J. 25295

28. Rangarajan S K 1974 J. Electroanal. Chem. 5627 